\title{
CCR7 and IRF4-dependent dendritic cells regulate lymphatic collecting vessel permeability
}

\author{
Stoyan Ivanov, ${ }^{1}$ Joshua P. Scallan, ${ }^{2}$ Ki-Wook Kim, ${ }^{1}$ Kathrin Werth, ${ }^{3}$ Michael W. Johnson, ${ }^{1,4}$ Brian T. Saunders, ${ }^{1}$ \\ Peter L. Wang, ${ }^{1}$ Emma L. Kuan, ${ }^{5}$ Adam C. Straub, ${ }^{6}$ Melissa Ouhachi, ${ }^{7}$ Erica G. Weinstein, ${ }^{5}$ Jesse W. Williams, ${ }^{1}$ \\ Carlos Briseño, ${ }^{1}$ Marco Colonna, ${ }^{1}$ Brant E. Isakson, ${ }^{6}$ Emmanuel L. Gautier, ${ }^{1,7}$ Reinhold Förster, ${ }^{3}$ Michael J. Davis, ${ }^{2}$ \\ Bernd H. Zinselmeyer, ${ }^{1}$ and Gwendalyn J. Randolph ${ }^{1}$ \\ 'Department of Pathology and Immunology, Washington University School of Medicine, St. Louis, Missouri, USA. ²Department of Medical Pharmacology and Physiology, University of Missouri, \\ Columbia, Missouri, USA. Institute of Immunology, Hannover Medical School, Hannover, Germany. ${ }^{4}$ Division of Biostatistics, Washington University School of Medicine, St. Louis, Missouri, USA. \\ ${ }^{5}$ Immunology Institute, Icahn School of Medicine at Mount Sinai, New York, New York, USA. ${ }^{6}$ Robert M. Berne Cardiovascular Research Center, University of Virginia School of Medicine, \\ Charlottesville, Virginia, USA. 'IINSERM UMRS 1166, Sorbonne Universités, University Pierre and Marie Curie (UPMC), University of Paris 06, Pitié-Salpêtrière Hospital, Paris, France.
}

\begin{abstract}
Lymphatic collecting vessels direct lymph into and from lymph nodes (LNs) and can become hyperpermeable as the result of a previous infection. Enhanced permeability has been implicated in compromised immunity due to reduced flow of lymph and immune cells to LNs, which are the primary site of antigen presentation to T cells. Presently, very little is known about the molecular signals that affect lymphatic collecting vessel permeability. Here, we have shown that lymphatic collecting vessel permeability is controlled by CCR7 and that the chronic hyperpermeability of collecting vessels observed in Ccr7 $7^{-1-}$ mice is followed by vessel fibrosis. Reexpression of CCR7 in DCs, however, was sufficient to reverse the development of such fibrosis. IFN regulatory factor 4-positive (IRF4+) DCs constitutively interacted with collecting lymphatics, and selective ablation of this DC subset in Cd11c-Cre Irf4//fl mice also rendered lymphatic collecting vessels hyperpermeable and fibrotic. Together, our data reveal that CCR7 plays multifaceted roles in regulating collecting vessel permeability and fibrosis, with one of the key players being IRF4-dependent DCs.
\end{abstract}

\section{Introduction}

DCs in the immune system are well characterized as potent antigenpresenting cells (APCs) for driving T cell-mediated host defense. Much of this activity takes place in lymph nodes (LNs), specialized filters of the immune system that are interspersed along lymphatic vessels that carry fluid, macromolecules, and recirculating cells from tissue interstitial space, with eventual return of the filtrate to the blood (1). Indeed, lymph is the major source of antigen supply to LNs, arriving either within migratory DCs traveling through lymph (1) or as free antigen available for capture by resident DCs already in LNs (2). Beyond antigen presentation, migratory DCs have been identified as critical players in governing functional homeostasis of the LN itself, acting to promote appropriate differentiation of high endothelial venules (HEVs) (3) and survival of stromal cells in the LNs (4). For any of these tasks, these cells travel to the LNs using the canonical G protein-coupled homing receptor CCR7. CCR7 is crucial to DC and T cell homing to LNs (5) and participates in the entry of DCs into lymphatic capillaries within peripheral organs such as skin and separately regulates the passage of LN-resident DC subsets across HEVs (6). The CCR7-dependent migration of DCs through the afferent lymphatic vasculature, however, dominates in its importance to the establishment of mature, functional

Authorship note: S. Ivanov and J.P. Scallan contributed equally to this work. Conflict of interest: The authors have declared that no conflict of interest exists. Submitted: September 1, 2015; Accepted: February 9, 2016. Reference information: / Clin Invest. 2016;126(4):1581-1591. doi:10.1172/JCI84518.
LNs. That is, if DCs fail to traverse the lymphatic capillary vasculature due to the lack of CCR7, the LNs does not acquire the appropriate characteristics to recruit $\mathrm{T}$ cells (7).

A major ligand for CCR7 is CCL21, constitutively expressed by lymphatic capillary endothelium and bound in a haptotactic gradient to aid in the guidance of DCs to the blind-ended lymphatic termini (8), where DCs enter through holes in the basement membrane and partially open flaps in the lymphatic vessel (9) without requirement for integrins to directly interact with lymphatic endothelium (10). Whether a DC perceives the CCL21 gradient is largely a function of whether it has matured sufficiently to express an appropriate amount of CCR7.

Although DCs appear to exclusively access the lymphatic system along the capillary portion of the lymphatic vasculature (9), close to lymphatic termini, the functional units of the lymphatic vasculature that ultimately control lymph flow are the larger lymphatic trunks, or lymphatic collecting vessels, that are downstream of lymphatic capillaries and receive input from them (1). Lymphatic collecting vessels possess intrinsic pumping activity that creates and sustains flow of lymph through the lymphatic system $(11,12)$ and, by extension, flow of interstitial fluid through interstitial spaces between cells in all organs (1).

Lymphatic collecting vessels are housed in adipose tissue around LNs. We have recently shown that DCs and macrophages that reside in this adipose tissue continuously sample lymph that, due to the basal permeability of lymphatic collecting vessels (13), leaks out into the surrounding perinodal adipose tissue (14). Only 
A

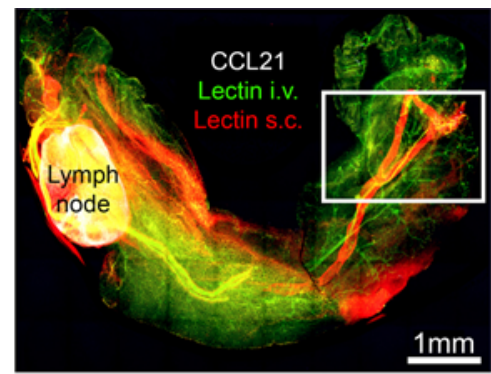

B

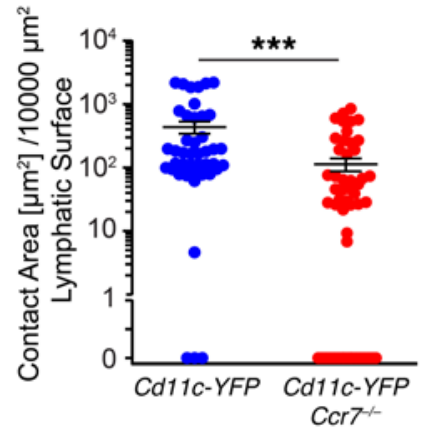

D

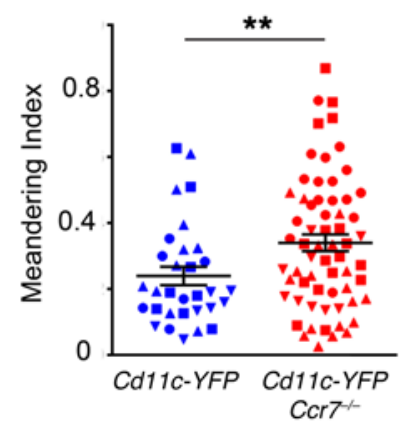

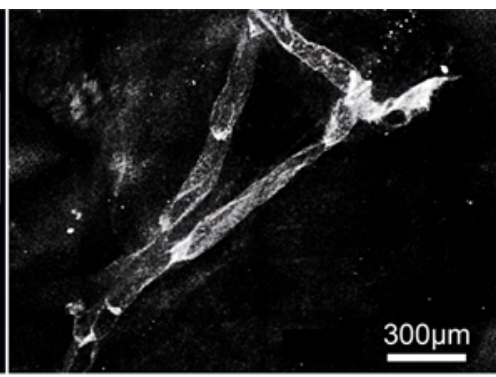

C

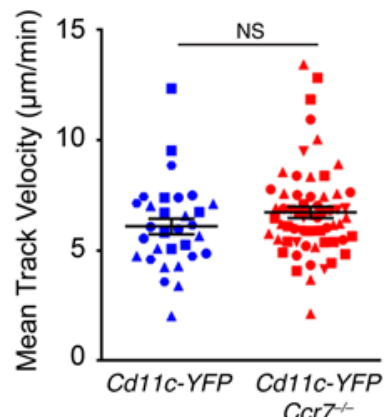

E

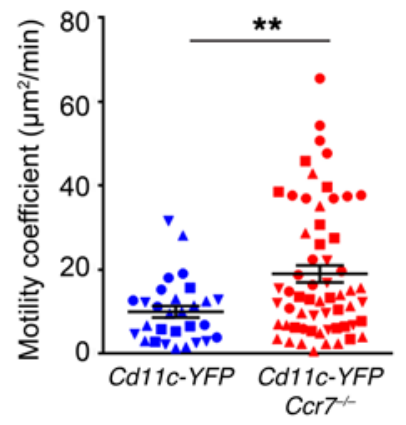

Figure 1. CCR7-mediated interactions of DCs with lymphatic collecting vessels outside of LNs. (A) Whole-mount preparation of brachial adipose tissue containing the brachial LNs with CCL21 staining shown in white. DyLight 594-conjugated lectin (red) was injected s.c. to visualize the draining lymphatic vasculature, and DyLight 488-conjugated lectin was i.v. administered in order to map the blood vasculature. White inset is placed around the major lymphatic collecting vessel afferent to the brachial LNs and shown at greater magnification on the right. (B) Contact area between DCs and lymphatic collecting vessels in $\mathrm{Cd11c-YFP}\left[\mathrm{Cr} 7^{+/+}\right.$and Cd11c-YFP $\left[\mathrm{cr} 7^{-/-}\right.$mice was quantified from single videos prepared during 2-photon intravital imaging, as described in Methods (see also Supplemental Video 2). Each data point represents analysis of 1 Z-stack, with 5 to 7 Z-stacks examined per mouse. Other measurements assessed from these videos were mean $\mathrm{DC}$ velocity (C), meandering index (D), and motility coefficient (E). From 4 to 5 mice per genotype were examined, with multiple areas along the lymphatic collecting vessel analyzed per mouse. For $\mathbf{C}-\mathbf{E}$, each data point represents tracking from an individual cell, with 7 to 10 cells tracked from 4 to 5 mice per genotype. In $\mathbf{C}-\mathbf{E}$, each mouse is plotted using a distinct symbol shape. Mann-Whitney $U$ tests were performed for assessment of statistical significance. ${ }^{* *} P<0.01 ;{ }^{* *} P<0.001$.

under exceptional circumstances do these lymphatic-associated, adipose tissue DCs mobilize to LNs (14), so the reason why they constitutively interact with the lymphatic collecting vessel and monitor the filtrate from this vessel has been unclear. Recently, extremely permeable lymphatic collecting vessels were identified as a consequence of aberrant healing following infection with Yersinia pestis (15). These collecting vessels became leaky enough to prevent the arrival of migratory DCs in draining mesenteric LNs. Consequently, immune protection was defective. The mechanisms accounting for the high permeability in lymphatic collecting vessels following $Y$. pestis infection remain unclear. Indeed, very little is known about how lymphatic permeability is regulated under any circumstance.

Here, we reveal a role for CCR7 in controlling lymphatic collecting vessel permeability. CCR7 deficiency leads to greatly increased basal permeability of lymphatic collecting vessels. At least part of the role of CCR7 in controlling this permeability relates to a role for DCs. In particular, the subset of DCs that intimately interacts with collecting vessels, IFN regulatory factor 4dependent (IRF4-dependent) CD11b $\mathrm{b}^{+} \mathrm{DCs}$, is implicated in the control of lymphatic permeability.

\section{Results}

Ccr $7^{-1-}$ mice exhibit fibrotic lymphatic collecting vessels and disrupted interactions with DCs. In contrast with lymphatic capillaries, lymphatic collecting vessels have not been observed as functioning as major sites of immune cell entry. Yet we observe that lymphatic collecting vessels constitutively displayed CCL21 (ref. 14 and Figure 1A). As DCs had also been observed to interact with collecting vessels (14), we wondered whether their interaction with the lymphatic collecting vessel might involve the CCL21 ligand CCR7. We thus crossed Cd11c-YFP (where YFP indicates yellow fluorescent protein) transgenic mice (16), in which intense YFP expression in perinodal adipose tissue marks DCs but not macrophages (14), with $\mathrm{Ccr}^{-/-}$and $\mathrm{Ccr} 7^{+/+}$mice to study this question. In some experiments, the progeny from this cross were further bred with Prox1ERT2-Cre (17) $\times$ Tomato reporter mice so that lymphatic vessels were constitutively fluorescent. $\mathrm{YFP}^{+}$DCs visualized in $\mathrm{Ccr}^{+/+}$ or $\mathrm{Ccr}^{7^{+-}}$mice intimately interacted with lymphatic collecting vessels, as has been previously published (14), and remained durably associated with them (Supplemental Videos 1-3; supplemental material available online with this article; doi:10.1172/ JCI84518DS1). Cytoplasmic extensions from DCs were observed to cross the lymphatic collecting vessel wall toward the lumen (Supplemental Video 1, $\mathrm{Crr}^{+/+}$analysis on left). DC motility around the vessel, and throughout the adipose tissue, remained high even when the lumen was not filled with tracers injected in the skin, but was instead made visible by Prox1-driven Tomato expression (Supplemental Video 2). DC activity was observed throughout the vessel area, including around valves (Supplemental Video 3). In contrast, $\mathrm{YFP}^{+}$DCs visualized in the $\mathrm{Ccr}^{-/-}$back- 
A

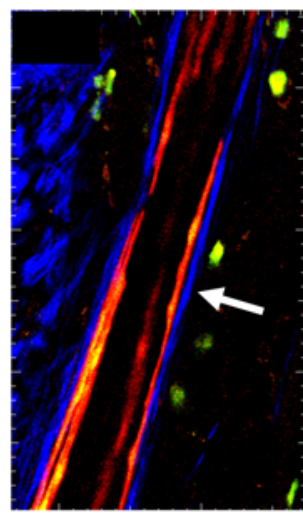

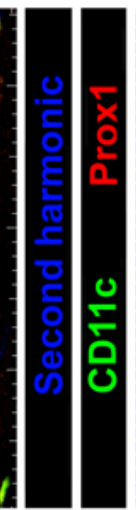
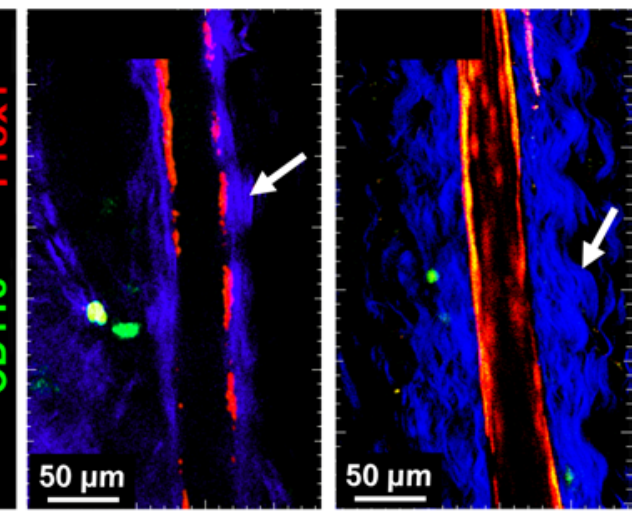

B

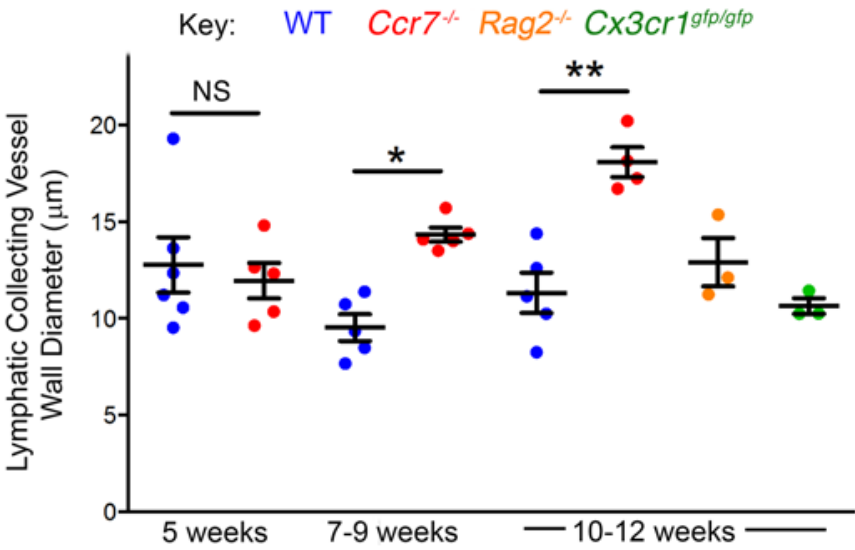

Figure 2. Increased adventitial wall thickness in lymphatic collecting vessels of $\mathrm{Ccr} \mathbf{7}^{-/-}$mice. (A) Images, each a single Z-plane, of the lymphatic collecting vessel afferent to the popliteal $\mathrm{LNs}$ in $\mathrm{CCr}^{+/-}$(left) or $\mathrm{CCr}^{-/-}$( 2 images on right) mice. White arrows point to the second harmonic signal. Scale bars: $50 \mu \mathrm{m}$. Image from $\mathrm{Ccr}^{-/-}$mouse in the middle is representative of the average difference compared with control mice; image on the far right displays the occasional regions of extreme fibrosis observed. (B) 2-Photon intravital analysis of the lymphatic collector vessel adventitial wall thickness in multiple strains of mice. From 3 to 6 mice per genotype were examined at multiple areas along the lymphatic collecting vessel analyzed per mouse. Each data point represents the mean result from 1 mouse after analysis of randomly selected time points from 2-photon images with 3 to 5 Z-stacks examined per mouse. For statistical evaluation, 1-way ANOVA with Tukey's post hoc test was carried out. ${ }^{*} P<0.05 ;{ }^{* *} P<0.01$. ground only briefly interacted or failed to interact with collecting vessels (Supplemental Video 1, $C c r 7^{-/-}$analysis on the right). We thus developed a method using MATLAB to quantify the surface of lymphatic vessels that were in contact with DCs (Supplemental Video 4 ) in the 2 genotypes. This analysis indicated an approximate 5-fold reduction in DC contact with collecting lymphatics in the $\mathrm{Ccr}^{-/-}$background (Figure 1B). Mean track velocity of DCs in $\mathrm{Ccr}^{-/-}$mice was not significantly different than in $\mathrm{Ccr}^{+/+}$ mice (Figure 1C). However, the meandering index, which assesses straightness of the migration path (a straight path is low meandering) and the aggregated motility coefficient, which assesses the net distance traveled from the point of origin, were higher in CD11c-YFP ${ }^{+}$DCs lacking CCR7 (Figure 1, D and E). Thus, in adult mice, absence of CCR7 expression impairs DC interaction with lymphatic collecting vessels in perinodal adipose tissue.

Examination of these videos, and analysis of individual Z-stacks from them, revealed not only reduced interaction of DCs with lymphatic collecting vessels in $\mathrm{Ccr}^{-/-}$mice, but also that the vessels possessed an increased second harmonic signal on the outside at the adventitial interface (Figure 2A; compare second harmonic wall diameter in the 2 videos included in Supplemental Video 1). We quantified the lymphatic adventitial diameter (thickness of region along the outer margins of the vessels corresponding to second harmonic signal) (Figure 2B). Collecting vessels in $\mathrm{Ccr}^{-/-}$mice displayed no differences from those of controls in the adventitial wall thickness at 5 weeks of age, but increased adventitial wall thickening was apparent by 7 to 9 weeks of age in $\mathrm{Ccr}^{7^{-1}}$ mice that was even more marked at 10 to 12 weeks of age (Figure 2B). Thickening of the lymphatic collecting adventitial wall was not recapitulated in Rag2 $2^{-/-}$mice lacking lymphocytes (Figure 2B), suggesting that adventitial wall thickening was not driven by a loss of lymphocyte interactions. This was an important issue since, besides DCs, lymphocytes depend upon CCR7 for trafficking. We also tested to determine whether other chemokine receptors on DCs gave rise to similar outcomes. DCs around lymphatic collecting vessels are from the CD11 $\mathrm{b}^{+}$subset of DCs (14) and express GFP in $\mathrm{C} x 3 \mathrm{cr}^{1 \mathrm{gp} /+}$ mice (Supplemental Figure 1), similarly to some other CD11b+ DCs (18). However, loss of CX3CR1 expression did not affect accumulation of the second harmonic signal in the lymphatic adventitia (Figure 2B).

Second harmonic signal in 2-photon microscopy is typically considered as deriving from accumulated collagen. To verify that such signal indeed corresponded to increased collagen in the adventitia of lymphatic collecting vessels from $\mathrm{Ccr}^{-/ /}$mice, transmission electron microscopy of lymphatic collecting vessels in $\mathrm{Crr}^{+/+}$and $\mathrm{Crr}^{-/-}$ perinodal adipose tissue was carried out. $\mathrm{Ccr} 7^{-/}$lymphatic collecting vessels displayed uneven masses of collagen fibrils outside of the vessels, which was especially evident at lower magnification (Figure 3, A and C). Fibroblasts were also observed within the excess adventitial collagen observed in $\mathrm{Ccr}^{-/-}$mice (Figure 3, compare A to $\mathrm{C}, \mathrm{B}$ to D). At higher magnification, individual collagen fibrils were discernible (Figure 3, E and F). These fibrils were denser and had a distinct appearance in $\mathrm{Ccr}^{-/-}$collecting lymphatics (Figure 3, E and F). This analysis confirms that the increased second harmonic generation quantified in $\mathrm{Ccr}^{-/-}$mice compared with $\mathrm{Crr}^{+/+}$mice (Figure 2) relates to an accumulation of extracellular matrix around the $\mathrm{Ccr}^{7-/}$ vessels. Overall, the data indicate lymphatic collecting vessel fibrosis occurs progressively in mice lacking CCR7. 

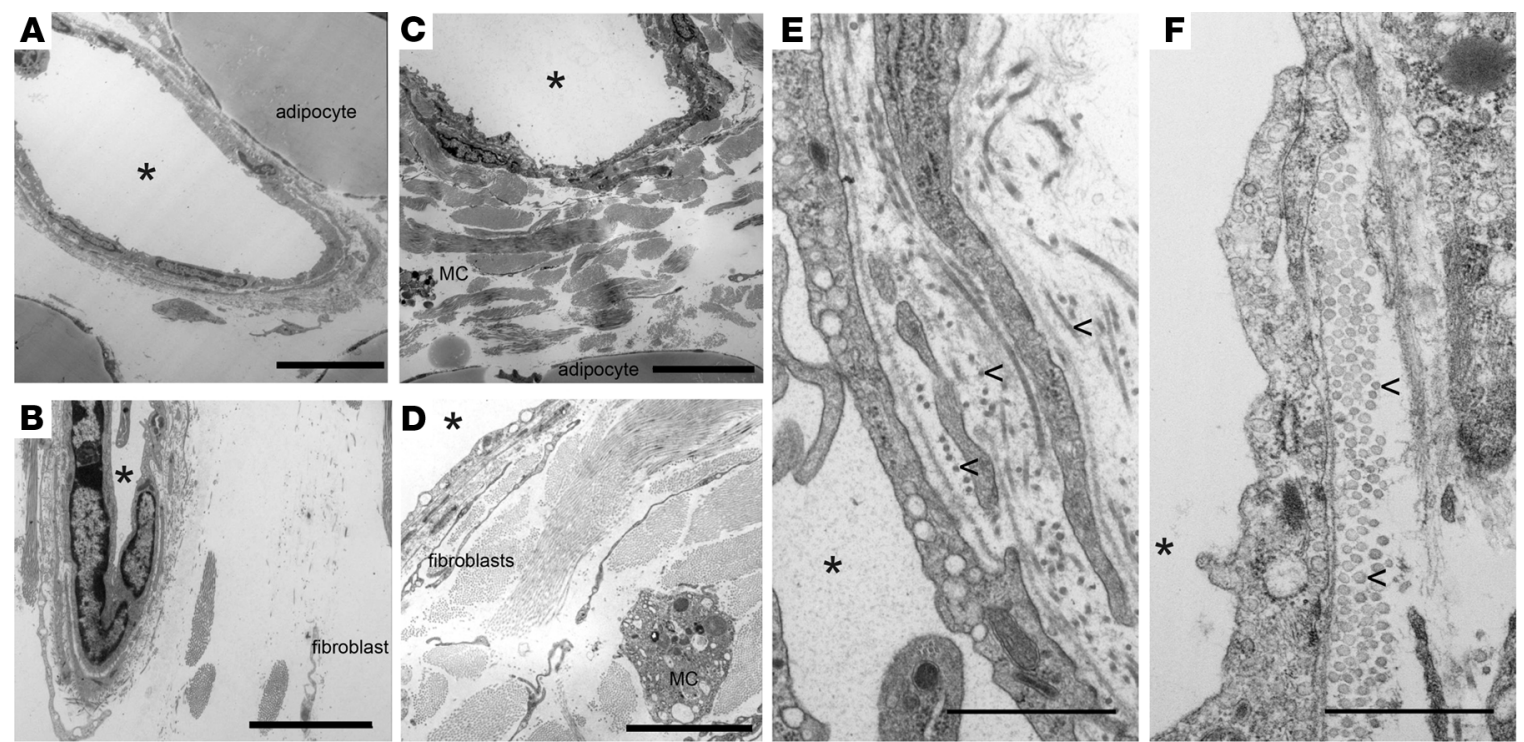

Figure 3. Electron micrographs of lymphatic collecting vessels within afferent s.c. adipose tissue near the brachial $\mathrm{LNs}$ from $\mathrm{Ccr} 7^{+/+}$or $\mathrm{Ccr} 7^{-/-}$mice. Lymphatic collecting vessels afferent to the brachial LNs of 12 -week-old $C \mathrm{Cr} 7^{+/+}$(A, B, and $\left.\mathbf{E}\right)$ or $\left[\mathrm{cr} 7^{-/-}\right.$(C, D, and F) mice were examined by transmission electron microscopy. Lumens of the collecting vessels are marked with asterisks, and representative fibroblasts (portions of their long and thin cytoplasmic projections), adipocytes, and mast cells (MC) are also labeled. Lymphatic endothelial cells are not labeled, but correspond with the cell type nearest the lumen marked with an asterisk. Matrix accumulation along the ablumenal or adventitial surface of the endothelium is relatively scarce in $\left[c r 7^{+/+}\right.$ samples (A and B), but far more evident in $\left[\mathbf{C r} 7^{-/-}\right.$tissues (C and $\mathbf{D}$ ). In $\mathbf{C}$, this matrix is observed between the lymphatic lumen and the adipocyte in the lower surface of the panel. In $\mathbf{D}$, the field of extracellular matrix occupies the vast majority of the image. In $\mathbf{E}$ and $\mathbf{F}$, representative fibrils of collagen are labeled with arrowheads. Scale bars: 10 microns ( $\mathbf{A}$ and $\mathbf{C}) ; 5$ microns (B and $\mathbf{D}) ; 1$ micron (E and $\mathbf{F})$. Six mice per genotype were examined over the course of 2 independent experiments.

Ccr $7^{-/-}$mice exhibit hyperpermeable lymphatic collecting vessels and loss of lymphatic transport from the periphery. Adding to the known impact of CCR7 deficiency on LN organization (7), the findings above suggested that lymphatic vessels supplying LNs also depend upon CCR7 to remain normal. We thus evaluated whether the clearance of tracer dye injected into ear skin, removed through a lymphatic vessel-dependent process (19), was altered in $\mathrm{Ccr}^{-/-}$mice compared with $\mathrm{Ccr}^{7^{+/}}$control mice. Indeed, clearance of Cy5-dextran from the ear was reduced by half in $\mathrm{Ccr}^{-/-}$mice (Figure 4A), indicating that lymphatic transport dysfunction was present in $\mathrm{Ccr}^{-/-}$mice. Because transport into lymph is ultimately regulated by the functionality of the downstream lymphatic collecting vessel $(11,12)$, we next evaluated functional properties of lymphatic collecting vessels in $\mathrm{Ccr}^{7--}$ mice. To this end, mouse lymphatic collecting vessels afferent to the popliteal LNs were exteriorized and investigated ex vivo (20). This procedure removes external adipocytes, but past studies have shown that DCs associated with the collecting vessels remain associated with them during such preparation (14). Lymphatic contractile function over a range of pressures was unaffected (Supplemental Figure 2). However, the permeability of lymphatic collecting vessels was markedly enhanced in $\mathrm{Ccr}^{-/-}$lymphatic collecting vessels compared with age-matched WT vessels from mice at 8 weeks or older (Figure 4B). Thus, we conclude that lymphatic collecting vessel permeability is regulated by CCR7.

Given the observation that permeability of lymphatic collecting vessels was markedly increased in $\mathrm{Ccr}^{-/-}$mice, we next hypothesized that (a) other changes in lymphatic collecting vessels observed in $\mathrm{Crr}^{-/-}$mice, notably fibrosis, might be secondary to the change in permeability and (b) that, given the robust interaction we observed between collecting vessels and DCs (Figure 1 and ref. 14), the role of CCR7 in controlling permeability involves a requirement for DCs in regulating lymphatic permeability. Support for the first hypothesis was obtained by the demonstration that, whereas fibrosis is a progressive phenotype in $\mathrm{Ccr}^{-/-}$mice not observed until 8 weeks of age, we found that the permeability was pervasively elevated, that is, we were able to cannulate lymphatic collecting vessels from mice as young as 5 weeks of age, and their permeability was elevated in these young mice similarly to the way it was in the older mice (Figure 4C). Therefore, hyperpermeability occurs prior to the fibrosis and is not a consequence of it. Other reports support the concept that fibrosis may be a consequence of increased flow across endothelium that would be induced by hyperpermeability $(21,22)$.

In support of the second hypothesis that DCs may use CCR7 to control permeability, we employed conditionally proficient $\mathrm{Ccr} 7$ gene-targeted mice in which the human CCR7 gene is knocked into the mouse $C c r 7$ locus, but is unable to be transcribed due to the presence of loxP sites flanking a neo insertion sequence (7). This mouse has been referred to as $C \mathrm{cr} 7^{-/-} C C R 7^{\text {stop } / \text { stop }}$. Conditional expression of CCR7 is possible in this strain when it is crossed with a strain in which Cre recombinase drives the removal of the neo cassette and thus expression of human CCR7 (7). Use of a Cd4-Cre driver restores CCR7 in $\mathrm{CD}^{+}$and $\mathrm{CD} 8^{+} \mathrm{T}$ cells ( $\mathrm{T}-\mathrm{Ccr} 7^{+/+}$mice), and CD11c-Cre driver restores CCR7 in DCs (DC-Ccr $7^{+/+}$mice) (7). Analysis of fibrosis in $\mathrm{Ccr}^{-/-} C C R 7^{\text {stopstop }}$ mice recapitulated that observed in $\mathrm{Ccr}^{-/-}$mice, where the second harmonic signal was elevated around collecting lymphatics (Figure 4D). Remarkably, 
A

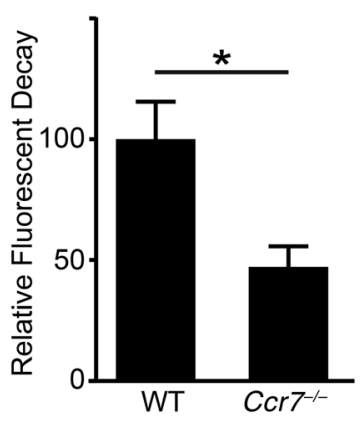

B

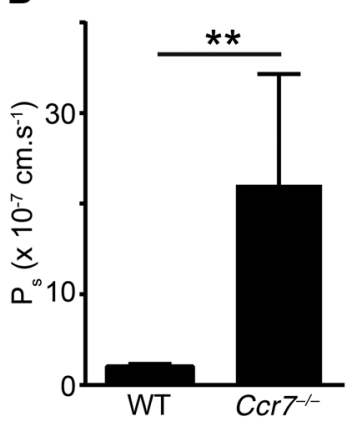

C

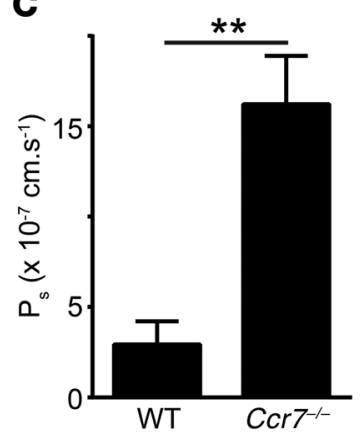

Figure 4. Lymphatic transport and permeability in CCR7deficient mouse strains. (A) $1 \mu$ l of Cy5-conjugated dextran $(70 \mathrm{kDa})$ was injected in the ear pinnae of anesthetized mice (5 to 10 weeks old), and the decrease of fluorescence intensity associated with its transport from the initial site of deposition was assessed using a fluorescence stereoscope ( $n=12 \mathrm{WT}, 7 \mathrm{Ccr}^{-1-}$ mice, mean \pm SEM). (B) Measurement of lymphatic collecting vessel permeability from mesenteric and popliteal adipose tissue in WT and $\mathrm{Crr}^{-1-}$ mice ( $n=5 \mathrm{WT}$ and $8 \mathrm{Ccr}^{-1-}$ mice combined from 2 independent experiments with mice at 10 to 12 weeks of age, mean \pm SEM). (C) Measurement of the lymphatic collecting vessel permeability in 5-week-old WT and Ccr7 $7^{-1-}$ mice ( $n=5 \mathrm{WT}$ and 7 Ccr7 ${ }^{-1-}$ mice, mean \pm SEM). (D) 2-Photon intravital analysis of adventitial wall thickness, assessed through measurement of second harmonic signal, around lymphatic collecting vessels in mice in which the human $C C R 7$ gene has been used to disrupt the mouse $\left[C r 7\right.$ locus (CCR $7^{\text {stop/stop }}$ ) or mice in which human CCR7 is expressed selectively on $\mathrm{CD}^{+} \mathrm{T}$ cells $\left(\mathrm{T}-\mathrm{CCr} 7^{+/+}\right)$or $\mathrm{CD} 11 \mathrm{c}^{+} \mathrm{DCs}\left(\mathrm{DC}-\mathrm{CCr} 7^{+/+}\right)$. In control mice, the $\mathrm{Ccr} 7$ gene is not disrupted (Total- $\left[\mathrm{Cr} 7^{--}\right.$). Six mice per genotype were examined at multiple areas along the lymphatic collecting vessels. Each data point represents the mean wall thickness of 1 mouse after blind scoring of 9 Z-stacks per mouse. For statistical evaluation, data in $\mathbf{A}-\mathbf{C}$ were assessed using the Mann-Whitney $U$ test and data in D employed 1-way ANOVA test, with Tukey's post hoc test. ${ }^{*} P<0.05 ;{ }^{* *} P<0.01$.

conditional expression of CCR7 that was limited to DCs (CD11 $\left.\mathrm{c}^{+}\right)$ was sufficient to prevent fibrosis in the collecting lymphatic vasculature (Figure 4D). Furthermore, there was a trend in the data suggesting that $\mathrm{T}$ cell reexpression of CCR7 was also able to reverse fibrosis, although this fell short of reaching statistical significance in our analysis. In relation to our hypothesis that CCR7-competent DCs prevent fibrosis, these data indicate that the expression of CCR7 by DCs indeed prevents fibrosis, but because T cells may be capable of preventing fibrosis as well if they are allowed to appropriately express CCR7, these data are best interpreted as showing that $\mathrm{CCR} 7^{+} \mathrm{DCs}$ are sufficient for preventing fibrosis, but it is less clear here if the DCs are necessary. One additional caveat in this scenario might be that some DCs express CD4 (23), while some $\mathrm{CD} 8^{+} \mathrm{T}$ cells express CD11c (24).

Lymphatic collecting vessel permeability is controlled by IRF4dependent DCs. Based on the findings above, we set out to further evaluate the role of DCs in controlling lymphatic vessel hyperpermeability and temporally downstream fibrosis. To assess whether the increased permeability of the lymphatic collecting vessels isolated from $\mathrm{Ccr}^{-/}$mice might be due to loss of DC interaction with the vessel, we depleted DCs in Cd11c-DTR transgenic mice (25) using diphtheria toxin (DT) in an attempt to disprove the hypothesis that DCs were regulators of permeability. As expected from previous studies (25), 1 dose of DT in Cd11c-DTR mice completely depleted perinodal adipose DCs for at least 36 hours (Supplemental Figure 3). We carried out DT-mediated depletion in Cd11c-DTR mice and compared them with DT-treated BDCA2-DTR mice as a DT treatment and depletion control to remove a different subset of DCs, the plasmacytoid DCs (26). The use of DT in BDCA2DTR mice allowed us to test whether DT-mediated killing led to nonspecific effects that affected permeability. Plasmacytoid DCs are the most populous DC subset in LNs, accounting for well over $50 \%$ of all LN DCs (27), and therefore we were able to investigate whether widespread LN DC depletion prior to lymphatic collecting vessel isolation was able to alter permeability. DT treatment in BDCA2-DTR mice gave rise to results consistent with those of WT mice without treatment (Figure 5A, compared with Figure $4, \mathrm{~B}$ and C). However, when conventional DCs were depleted in Cd11c-DTR mice 36 hours after administering DT and then lymphatic collecting vessels were isolated for ex vivo studies, permeability was increased more than 4 -fold (Figure 5A). This result is consistent with the possibility that DCs acutely regulate lymphatic collecting vessel permeability. We also determined whether acute depletion of DCs using DT treatment in Cd11c-DTR mice led to a reduction in lymphatic transport of dyes injected into the ear pinnae. In this assay, we found no differences between mice with or without DCs (Figure 5B), indicating that the loss of lymphatic transport from the ear and increased permeability of collecting vessels were separable processes.

However, while the use of a DT-based system to study DC function is helpful, caveats are numerous (28). We thus searched for additional approaches to examine the importance of DCs more selectively. The transcription factor IRF4 has been linked to the presence of the CD11 $\mathrm{b}^{+}$subset of DCs in some tissues $(29,30)$. Given that DCs in perinodal adipose tissue are mainly of the $\mathrm{CD} 11 \mathrm{~b}^{+}$subset (14) and that those expressing CX3CR1 are CD11b+ and located around collecting vessels (Supplemental Figure 1), we examined whether perinodal adipose tissue $\mathrm{CD}_{11 \mathrm{~b}^{+}}$DCs were deleted in $\mathrm{Irf} 4^{-/}$mice or Cd11cCre Irf $4^{f / f l}$ mice. First, whole-mount immunostaining of perinodal adipose tissue around the brachial LNs revealed a partial reduction in local MHC $\mathrm{II}^{+}$cells in total body $\mathrm{Irft}^{-/-}$mice com- 
A

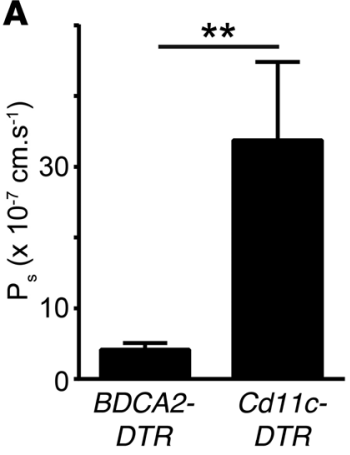

B

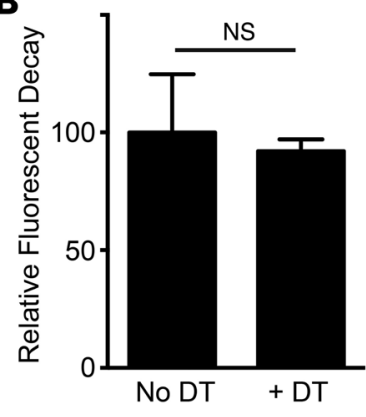

Figure 5. Evaluation of lymphatic permeability and transport in mice following acute DC depletion. (A) Cd11C-DTR and BDCA2-DTR mice were injected with DT at $4 \mathrm{ng} / \mathrm{g}$ of body weight; 36 hours after the administration of the toxin, the lymphatic collecting vessel was exteriorized and permeability was assessed ( $n=9$ mice per genotype, combined from 2 independent experiments, mean \pm SEM). (B) $1 \mu \mathrm{l}$ of Cy5-conjugated dextran (70 kDa) was injected in the ear pinnae of anesthetized mice, and the decrease of fluorescence intensity associated with its transport from the initial site of deposition was assessed using a fluorescence stereoscope 36 hours after the administration of DT at $4 \mathrm{ng} / \mathrm{g}$ of body weight ( $n=6$ control and 6 Cd11c-DTR + DT mice combined from 2 independent experiments, mean \pm SEM). For statistical evaluation, data were assessed using the Mann-Whitney $U$ test. ${ }^{* *} P<0.01$.

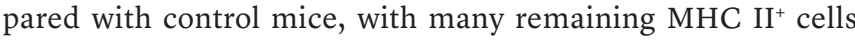
in the $\mathrm{Irf4}^{-/-}$mice exhibiting a rounded morphology (Supplemental Figure 4A). By flow cytometry, Irf4 ${ }^{-/}$mice indeed had a reduced number of $\mathrm{CD} 11 \mathrm{~b}^{+} \mathrm{DCs}$ in perinodal adipose tissue and associated LNs (Supplemental Figure 4, B and C). We followed up on these observations and generated Cd11c-Cre $\operatorname{Irf} 4^{f l / f l}$ mice, where deletion of Irf 4 would occur in all DCs, most nonclassical monocytes, and in a minority of $\mathrm{T}$ and $\mathrm{B}$ cells (31). As in previous studies (31), we eliminated the occasional mouse whose blood lymphocytes indicated a greater than $25 \%$ deletion in the $\mathrm{T}$ or $\mathrm{B}$ cell compartment, as reported by the expression of GFP downstream of Irf4 gene deletion (32). As in the lung and intestine (31), the CD11b+ DC subset was reduced by more than half in perinodal adipose tissue around skin-draining LNs or mesenteric adipose tissue (Figure 6, A and B), but the CD24+ subset of DCs that also express CD103 and depend upon BATF3 (33) was not reduced in the adipose tissue (Figure 6, A and B). In brachial LNs, total cellularity was normal (Figure 6C). Some subsets of the migratory DCs distinguished as a class by very high levels of MHC II (Figure 6D) were reduced in LNs, especially those that were $\mathrm{CD}_{11} \mathrm{~b}^{+}$(Figure 6E), but resident LN DCs were not significantly affected (Figure 6F). In mesenteric LNs, migratory DCs are recognized as $\mathrm{CD} 103^{+} \mathrm{CD} 11 \mathrm{~b}^{+}(15,34)$. These migratory DCs, as expected in Cd11c-Cre Irf $4^{f l / f l}$ mice (34), were significantly reduced in mesenteric LNs (Figure 6, G and $\mathrm{H}$ ), but the resident LN DCs and overall LN cellularity remained close to normal (Figure 6, $\mathrm{H}$ and I).

Having established reduced perinodal DCs, along with lymph-migratory LN DCs, in Cd11c-Cre $\operatorname{Irf} 4^{f / f l}$ mice, we next addressed whether the same lymphatic function parameters altered in $\mathrm{Ccr}^{-/-}$mice were affected. Indeed, lymphatic collecting vessel permeability was increased in lymphatic collecting vessels from the adipose tissue afferent to mesenteric or popliteal LNs in Cd11c-Cre Irf $4^{f l / f l}$ mice to a degree similar to that in $\mathrm{Crr}^{-/-}$mice (Figure 7A). However, lymphatic transport of tracers injected into ear pinnae of Cd11c-Cre $\operatorname{Irf} 4^{f / f l}$ mice was not significantly reduced (Figure $7 \mathrm{~B}$ ), leaving outcomes in this assay distinct from those in $\mathrm{Ccr}^{-/-}$mice (Figure 4A) and mirroring those of acute DC depletion in Cd11c-DTR mice (Figure 5, A and B). DC depletion in Cd11c-Cre Irf $4^{f l / f l}$ mice did not alter lymphatic capillary morphology significantly, whereas lymphatic capillaries were dilated in $\mathrm{Ccr}^{-/-}$mice (Supplemental Figure 5), suggesting that defective lymphatic capillaries may account for the poor transport in $\mathrm{Ccr}^{-1-}$ mice that remains intact in $\mathrm{Cd} 11 \mathrm{c}$ -
Cre Irf $4^{f l f l}$ mice. Finally, the more chronic loss of DCs in the state of IRF4 deficiency, as compared with that in Cd11c-DTR mice with an acute loss of DCs, allowed us to observe accumulation of collagen in the adventitial wall of collecting vessels in IRF4 deficiency (Figure 7C), thus supporting a strong relationship between chronically elevated lymphatic permeability and fibrosis of lymphatic collecting vessels.

\section{Discussion}

The functionality of the lymphatic collecting vessel that has spontaneous contractile properties to promote lymph flow has not historically been a concern in immunology. However, recently, it has become clear that the permeability of the collecting vessels can affect the broadcast of antigen to APCs (14). Moreover, leakage or extreme elevations in permeability, at least in the intestine and mesenteric lymphatic network, have been associated with failure of the downstream LNs to receive input from DCs that normally enter LNs via the upstream lymphatic vasculature, leading to failed immunity and persistent inflammation (15). Through these observations, Fonseca de Morais et al. highlighted the need to better understand the mechanisms that regulate permeability in lymphatic collecting vessels, of which very little is presently known, in order to control inflammation and immunity. This body of work brings forward the chemokine receptor CCR7, expressed in some immune cells (DCs, T and B cells, innate lymphocytes), but no other known cell types, as a molecule that strongly affects lymphatic collecting vessel permeability.

Specifically, we found that genetic deletion of $\mathrm{Cr} 7$ in mice elevates basal lymphatic collecting vessel permeability in mesenteric and skin-draining vessels by approximately 5 -fold. This increase in permeability was observed in mice of all ages examined, including those as young as 5 weeks, the youngest age at which we could quantify collecting vessel permeability due to technical constraints. In addition to chronically elevated permeability in $\mathrm{Crr}^{-/-}$mice, we observed collagen accumulation, apparent fibrosis, around the collecting vessel adventitia starting at 7 to 9 (Figure 2B) weeks of age, but not earlier. Based on previous literature, we propose that these fibrotic changes are an expected downstream consequence of persistently elevated flow across hyperpermeable vessels $(21,22)$. The inevitable onset of fibrotic changes, which were easily measured using 2-photon microscopy via measurement of the second harmonic signal, allowed us to extend our explorations on the role of the CCR7 beyond what we would be able to execute employing only the quite time-consum- 
A

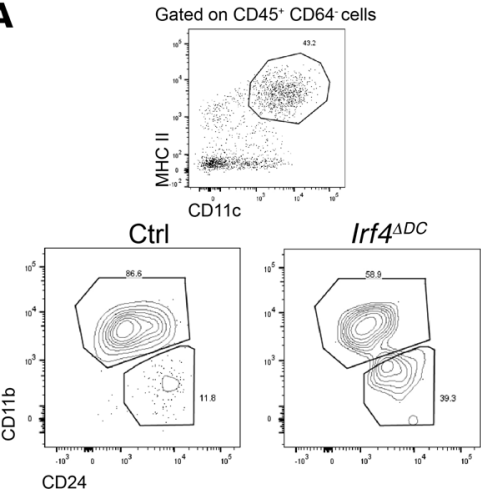

D

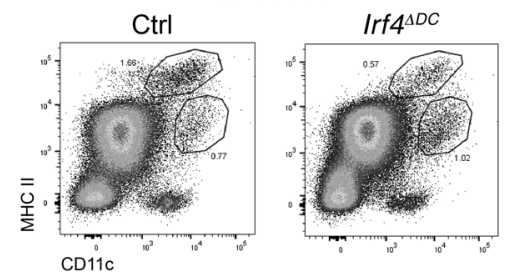

G

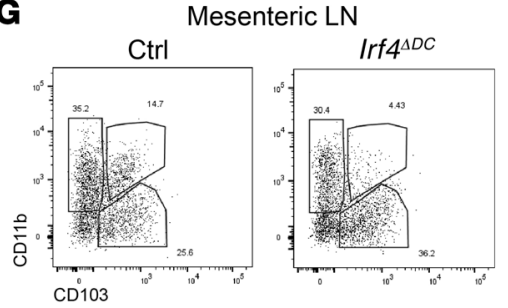

B

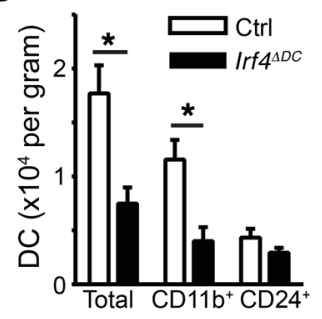

C

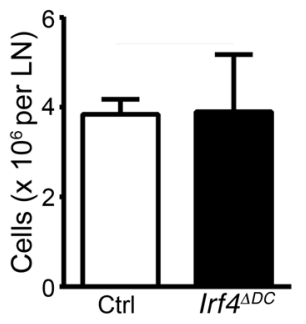

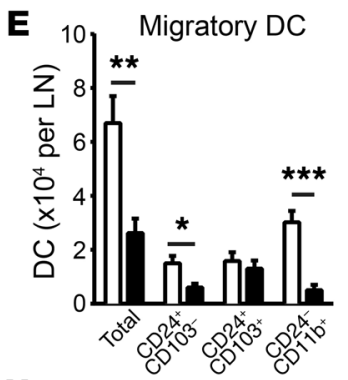

H

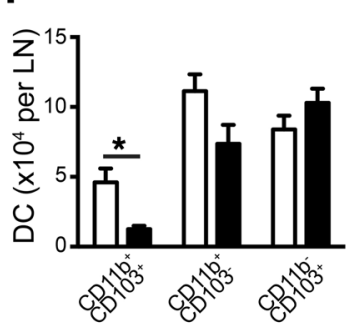

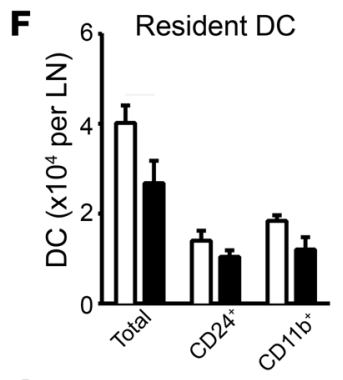

I

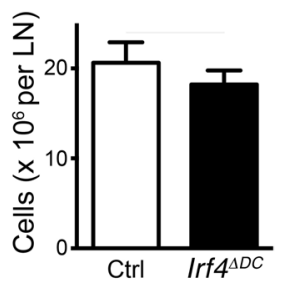

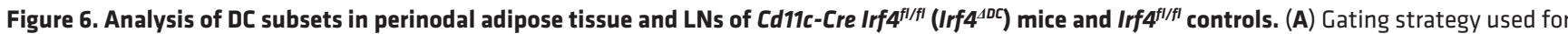
identification of adipose tissue DC subsets, with mesenteric adipose tissue shown after pregating on CD45 ${ }^{+} \mathrm{CD} 64^{+}$macrophages. DCs were identified as $\mathrm{CD}^{+} 5^{+} \mathrm{CD} 644^{-} \mathrm{MHC} \mathrm{II}{ }^{+} \mathrm{CD} 11 \mathrm{c}^{+}$(upper panel). Further, DCs were subdivided according to their cell-surface expression of the markers CD11b and CD24 (lower panel). (B) Enumeration of the DCs in the perinodal adipose tissue around brachial LNs ( $n=3$ for each genotype, mean \pm SEM). (C) Cellularity of the brachial LNs of control and Irf $4^{\triangle D C}$ mice $\left(n=5\right.$ for each genotype, mean \pm SEM). (D) Gating strategy used for identification of migratory (MHC II ${ }^{\text {hi }}$ and resident DCs in the brachial LNs. Quantification of the populations of migratory (E) and resident (F) DCs in the brachial LNs ( $n=5$ for each genotype, mean \pm SEM). (C) Gating strategy used for identification of DC subsets in the mesenteric LNs. DCs were pregated as MHC II+CD11c ${ }^{+}$cells and then gated into 3 populations as shown, including CD11b+CD103+ cells, which are migratory DCs that fall between 2 resident DC subsets. (H) Quantification of the populations of DCs in the mesenteric LNs ( $n=4-5$ for each genotype, mean \pm SEM). (I) Cellularity of the mesenteric LNs of control and Irf $4^{\Delta D C}$ mice $(n=4-5$ for each genotype, mean \pm SEM). For statistical evaluation, data were assessed using the Mann-Whitney $U$ test. ${ }^{*} P<0.05 ;{ }^{* *} P<0.01 ;{ }^{* *} P<0.001$. Ctrl, control.

ing permeability assay. In particular, we measured elevated adventitial second harmonic signal in a second CCR7-deficient strain in which the human CCR7 gene was inserted in the mouse $C c r 7$ locus downstream of a floxed neo cassette (7). Cre recombinase-mediated induction of CCR7 in DCs was sufficient to reverse the latestage fibrotic development around lymphatic collecting vessels. However, reexpression in $\mathrm{CD} 4^{+}$cells, mostly $\mathrm{T}$ cells, also strongly trended toward correction of the fibrotic phenotype, even though adventitial collagen was not increased in $\mathrm{Rag}^{-/-}$mice entirely lacking T cells. It is thus possible that the role of CCR7 in affecting lymphatic collecting vessel functionality can be supplied by multiple $\mathrm{CCR} 7^{+}$cell types, but the role for DCs seems especially clear.

In addition, because intravital imaging data revealed that DCs actively interact with lymphatic collecting vessels, we carried out further experiments to investigate the role of DCs in affecting lymphatic collecting vessel function. This analysis revealed that deletion of IRF4 selectively in $\mathrm{CD} 11 \mathrm{c}^{+}$cells reca- pitulated the increased permeability, and ultimately fibrosis, in lymphatic collecting vessels that was observed in $\mathrm{Crr}^{-1-}$ mice. Because IRF4 in DCs did not significantly reduce the presence of resident DCs in LNs, it is unlikely that resident LN DCs act as the regulators of collecting vessels. Instead, the data point to 2 potential DC populations that were greatly decreased in $\mathrm{Cd11c}$ Cre Irf $4^{f l / f l}$ mice and are more likely culprits: (a) CD11b ${ }^{+}$DCs that localize around collecting vessels, constitutively and intimately interacting with them, and (b) lymph-migratory DCs that home to LNs, which we confirmed, in agreement with the work of others (34), are greatly decreased in Cd11c-Cre Irf $4^{f / f l}$ mice. Unfortunately, there are no additional genetic tools or other approaches available at present to further refine our analysis to distinguish which of these DC populations is most relevant to control of afferent collecting vessel permeability. It might seem most logical that the DCs that physically contact the collecting vessel regulate their permeability. Fitting with that hypothesis are our 
A

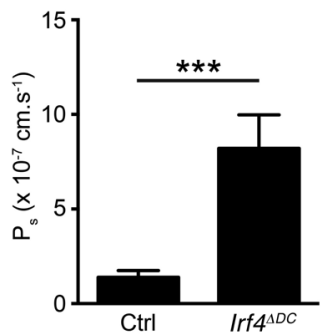

B

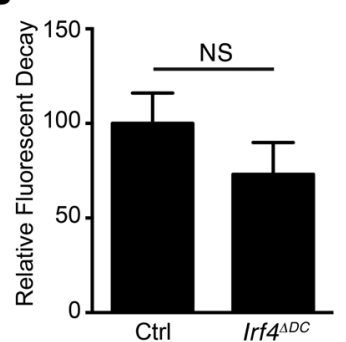

C

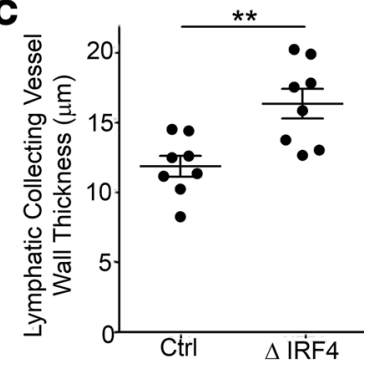

Figure 7. Analysis of the role of IRF4-dependent DCs in lymphatic transport and permeability. (A) Measurement of lymphatic collecting vessel permeability taken from mesenteric and popliteal adipose tissue of control (Ctrl; Irf $\left.4^{f / f}\right)$ and Irf4 $4^{\Delta D C}\left(C D 11 c\right.$-Cre Irf $\left.4^{f / f f}\right)$ mice $(n=4-5$ mice per genotype, mean \pm SEM). (B) $1 \mu$ of Cy5-conjugated dextran ( $70 \mathrm{kDa}$ ) was injected in the ear pinnae of anesthetized mice, and the decrease of fluorescence intensity associated with its transport from the initial site of deposition was assessed using a fluorescence stereoscope ( $n=7$ mice per genotype, mean \pm SEM). (C) 2-Photon intravital analysis of adventitial wall thickness, assessed through measurement of second harmonic signal, around lymphatic collecting vessels in control and Irf4-deficient mice (Irf4 $4^{-/-}$or Cd11c-Cre Irf $4^{f / f}$ ) . Eight mice per genotype were examined at multiple areas along the lymphatic collecting vessels, with multiple videos made in different areas per mouse. Each data point represents the mean of 1 mouse after blind scoring of 6 to 12 Z-stacks per mouse. For statistical evaluation, data in $\mathbf{A}$ and $\mathbf{B}$ were assessed using the Mann-Whitney $U$ test. ${ }^{*} P<0.01 ;{ }^{* *} P<0.001$. Data in C were assessed using 1-way ANOVA, Tukey's post hoc test.

data showing that isolated vessels, bearing no direct connection to LNs when prepared ex vivo, still show a DC-dependent shift in permeability. However, what key signals are provided by DClymphatic interactions on the collecting vessel route through adipose tissue remain unclear. $\mathrm{CD} 11 \mathrm{~b}^{+} \mathrm{DCs}$ isolated from adipose tissue do show selective expression of extracellular matrix components such as fibronectin and proteases such as PAI-1 (http:// www.immgen.org) that in other areas of vascular biology strongly affect vessel function. Future studies are needed to evaluate the role of these and other adipose DC-selective molecules in the regulation of lymphatic collecting vessel permeability.

It is also reasonable to consider that lymph-migratory DCs that pass through collecting vessels on their way to LNs control lymphatic collecting vessel function, although how they would do so in assays that utilize isolated vessels lacking LNs is unclear. Such lymph-migratory DCs, exquisitely dependent upon CCR7 for their trafficking to LNs, are known to regulate the maturation of the specialized postcapillary venules in LNs, the HEVs $(3,4,7)$. From this perspective, it is interesting to note that classical studies on lymphatic collecting vessels in dogs, done in vivo with LNs remaining functional during collecting vessel cannulations, show a key role for LN venules in fluid exchange and lymph flow (35-37). Specifically, the venules in LNs are responsible for uptake of water during the appreciable concentration of afferent lymph before it traverses the LN and enters efferent lymph (35). High pressure in the efferent lymphatic collecting vessels was able to drive afferent lymphatic vessel leakiness due to venous collapse in the LNs (37). Thus, a role for migratory DCs in controlling the maturation state and overall functionality of the HEVs may indeed influence the permeability of the afferent lymphatic collecting vessels that we have studied here, perhaps even after these vessels have been isolated and separated from LNs.
Bringing these concepts to pathophysiology, Fonseca de Morais et al. argued that dysfunctionality in afferent lymphatic collecting vessels prevented migratory DCs from reaching mesenteric LNs, thus setting up a state of dysregulated immunity that, in the intestine, might promote processes associated with inflammatory bowel disease (15). However, if migratory DCs control the functionality of lymphatic collecting vessels, as described above, the relationship might be the other way around: circumstances that ablate DC migration through lymphatics alter both the function of LNs and upstream lymphatics. The potential connection of these concepts to inflammation and disease makes this an important area for further future investigation.

Finally, while our live imaging experiments, specialized functional assays, and genetic analyses link DCs to regulation of collecting vessel physiology, our studies on CCR7 point to roles in lymphatic function that may involve cells other than DCs. Not only do our data hint that CCR7 expression on $\mathrm{T}$ cells may affect lymphatic collecting homeostasis, but the removal of fluorescent tracers from the ear interstitium via lymphatic capillary vessels was impaired in $\mathrm{Ccr}^{-/-}$mice, but not in mice with DCspecific ablation of IRF4. When we then visualized the lymphatic capillaries in skin, only those in $\mathrm{Ccr}^{-/-}$mice were dilated, consistent with fluid accumulation during impaired transport (38). Since both loss of IRF4 in DCs and CCR7 deficiency led to similarly altered lymphatic collecting vessel permeability, this transport defect in lymphatic capillaries of $\mathrm{Ccr}^{-1-}$ mice is likely unrelated to altered lymphatic permeability. In short, this work identifies roles for CCR7 in controlling lymphatic vessel transport and function, and a major player, though perhaps not alone, in this regulation is the IRF4 $4^{+}$DC. This insight will be useful in further dissecting the underlying pathophysiology following $Y$. pestis infection and beyond.

\section{Methods}

Animals. Cx3cr1 $1^{g f / g f p}$ mice and C57BL/6 WT mice were purchased from The Jackson Laboratory. $C c r 7^{-/}$mice (5) were purchased from The Jackson Laboratory and maintained at Washington University School of Medicine or bred and maintained at Hannover Medical School along with $C \mathrm{cr}^{-/-} C C R 7^{\text {stop/stop }}$ mice crossed or not to Cd11c-Cre or CD4Cre mice (7). $C \mathrm{Cr}^{-1-}$ mice were interbred with $C d 11 c-Y F P$ reporter mice (16). Cd11c-DTR transgenic mice, also purchased from The Jackson Laboratory, have been described (25), as have BDCA2-DTR mice bred and maintained at Washington University (26). Prox1-ERT2-Cre mice (17) were a gift from Guillermo Oliver (Northwestern University, Chicago, Illinois, USA) and were bred to Rosa26S or floxed-stop tdTomato (fstdT) reporter mice (The Jackson Laboratory, JAX stock number 007905). Resulting Prox1-ERT2-Cre Tomato reporter mice were utilized for imaging or further crossed to $C c r 7^{-/-} C D 11 c-Y F P$ reporter mice described above. Irf $4^{-/}$mice (39) were bred and maintained at Washington University and provided for these studies by Ken Murphy of that institution. Irf $4^{\triangle D C}$ mice were generated at Washington University 
by breeding B6.129S1-Irf4 $4^{\text {tmIRdf }} / \mathrm{J}\left(\operatorname{Irf} 4^{f / f l}\right)$ mice with B6.Cg-Tg(ItgaxCre)1-1Reiz/J (Itgax-Cre) mice (both strains from The Jackson Laboratory). This strain was exported, maintained, and additionally studied at the INSERM UMRS 1166 (Paris, France).

Immunostaining and whole-mount imaging. Tissues were fixed overnight in $4 \%$ PFA containing 30\% sucrose. Tissues were then incubated in $1 \%$ chicken OVA plus $0.015 \%$ Triton $\mathrm{X}-100$ for 2 hours or more, depending on the size of the tissue, and then transferred to PBS containing $0.2 \%$ OVA and primary Abs overnight at $4^{\circ} \mathrm{C}$. Immunostaining was carried out using anti-MHC II (eBioscience, clone M5/14.15.2), anti-CCL21 (R\&D Systems, clone 59106), or anti-podoplanin Ab (purchased from AngioBio). Three washes were carried out in PBS, at 10 minutes each, and then secondary, conjugated Abs were added at $4^{\circ} \mathrm{C}$ overnight. Secondary Abs used for detection of these Abs were Cy3conjugated anti-rat $\mathrm{Ab}, \mathrm{Cy5}$-conjugated anti-rabbit $\mathrm{Ab}$, and Cy5-conjugated anti-Syrian hamster $\mathrm{Ab}$ (both from Jackson Immunoresearch). Nuclear contrast was provided by the addition of DAPI. Washed tissues were then dehydrated in a series of alcohol dilutions that started with $70 \%$ ethanol and culminated in $100 \%$ ethanol. Then tissue was transferred into methyl salicylate to achieve optical clarification that permitted optimal confocal imaging. Images were acquired on a Leica SPE confocal microscope equipped with a tile-scanning feature.

Flow cytometry. Single-cell adipose tissue suspensions were obtained from minced adipose tissue subjected to collagenase D (3 $\mathrm{mg} / \mathrm{ml}$ ) (Roche) digestion for 45 minutes at $37^{\circ} \mathrm{C}$ and then treated with red blood cells lysis buffer (BD Biosciences). Cells were stained with combinations of the following mAbs for 25 minutes on ice, protected from light. mAbs included those directed against CD45 V500-conjugated Abs (BD Horizon, clone 30-F11) or Pacific Blue-conjugated Abs (BioLegend, clone 30-F11), CD11c Pe-Cy7-conjugated Abs (BioLegend, clone N418), CD11b APC-Cy7-conjugated Abs (Biolegend, clone M1/70), CD24 PerCP-Cy5.5-conjugated Abs (BD Biosciences - Pharmingen, clone M1/69), CD64 APC-conjugated Abs (BD Biosciences - Pharmingen, clone X54-5/7.1), F4/80 APC-Cy7-conjugated Abs (BioLegend, clone CI:A3-1), and MHC II eFluor450-or FITC-conjugated Abs (eBioscience, clone M5/14.15.2). Abs to MerTK (BAF 591) were from R\&D Systems and were used in combination with streptavidin PerCP-Cy5.5-conjugated Abs (BioLegend). Cells were analyzed on the LSR Fortessa (BD) instrument equipped with DIVA software. Samples were later analyzed using FlowJo software (Tree Star).

2-Photon microscopy. Mice were anesthetized by i.p. injection of ketamine $(50 \mathrm{mg} / \mathrm{kg})$ and xylazine $(10 \mathrm{mg} / \mathrm{kg})$ and maintained with halved doses administered every hour. Mice analyzed at Hannover Medical School received 1 i.p. injection of ketamine $(100 \mathrm{mg} / \mathrm{kg})$ and medetomidine $(1 \mathrm{mg} / \mathrm{kg})$. Mouse legs were carefully shaved with Nair (Church \& Dwight Co.) and washed with $1 \times$ PBS to remove the extra lotion. The superficial layer of the skin was removed, and the lymphatic collecting vessels in the popliteal region were visualized via s.c. injection of 10 to $20 \mu \mathrm{l}$ of DyLight 594-conjugated tomato lectin (Vector Laboratories) into the footpad. During the acquisition, mouse status was closely monitored.

Images were collected at Washington University using a customized Leica SP8 2-photon microscope equipped with a $\times 25 / 0.95$ NA water-dipping objective and a Mai Tai HP DeepSee Laser (SpectraPhysics) tuned to $900 \mathrm{~nm}$. Fluorescence emission was separated by 3 high-efficiency dichroic mirrors cutting at 458, 495, and $560 \mathrm{~nm}$ (Semrock) and directly directed to 4 supersensitive external detectors.
3D stacks consisting of between 21 and 31 planes ( $2.5 \mu \mathrm{m}$ step size) were captured every 30 seconds.

Alternatively, for 2-photon imaging at Hannover Medical School, the following microscope setup was used: TriM Scope (LaVision Biotec), Olympus BX51 upright microscope, ×20/0.95 water immersion objective (Olympus), and MaiTai Ti:Sa pulsed infrared laser (Spectra-Physics) at $865 \mathrm{~nm}$. For separation, this system used longpass filters at 485,550, and $600 \mathrm{~nm}$ and additional bandpass filters of $447 / 60$ and $624 / 40$. Z-stacks were acquired over a total of 31 to 71 planes ( $2-\mu \mathrm{m}$ step size).

2-Photon excitation produced a second harmonic signal from collagen within the analyzed tissue (40). Multidimensional rendering and manual cell tracking was done with Imaris (Bitplane). Mean track velocities $(\mu \mathrm{m} / \mathrm{min})$ and motility coefficients $\left(\mu \mathrm{m}^{2} / \mathrm{min}\right)$ were calculated for individual tracks spanning 11 time points (or 5 minutes) (41). The motility coefficient was defined as the slope of the fitted line of displacement squared versus time. The meandering factor was calculated by dividing the displacement of a cell by its total path length. All track calculations were performed using T cell Analyser version 1.7.0 software (http://spider.science.strath.ac.uk/sipbs/showPage.php?page=software_imaging), written by J. Dempster (University of Strathclyde, Glasgow, Scotland) (40). Data were transferred and plotted in GraphPad Prism 6 (GraphPad Software Inc.) for creation of the graphs.

Electron microscopy. WT and $C c r 7^{-1-}$ mice were euthanized and immediately perfused with $4 \%$ paraformaldehyde and $2.5 \%$ glutaraldehyde in PBS. Anterior s.c. fat containing brachial LNs was excised and cut in the midsection with respect to the region containing the afferent lymphatic vessel (14), and blocks were prepared and processed for standard transmission electron microscopy by the University of Virginia Advanced Microscopy and Histology Core. Sections were evaluated using a Joel 1220 electron microscope.

Analysis of adventitial wall diameter and luminal diameter of lymphatic collecting vessel. Single 2-photon-generated videos were analyzed at Washington University School of Medicine and Hannover Medical School by individuals blinded to the genotype and age of the mice used to generate the data. For analysis, multiple Z-stacks per mouse were examined in the middle region (along the $z$ axis) of the vessel, and the thickness of the lymphatic collector wall and the diameter of the lumen of the collector vessel were quantified using Imaris software. Further, the ratio between the lymphatic lumen diameter and the lymphatic wall diameter was calculated. After data were unblinded, graphical depictions of the results were prepared using GraphPad Prism 6.

Estimation of surface area of contact between lymphatic vessel and DCs. Surfaces of the lymphatic vessel and DCs were created using the surface function in Imaris. The surfaces were then imported into MATLAB as X, Y, and Z Cartesian coordinates. To determine the contact between the vessel and the cell, a threshold was predetermined (1 micron), and the distance between each vessel and cell Cartesian coordinate was calculated using the Euclidean distance formula (Equation 1). The points of the vessel that were within the distance threshold were then used to estimate the surface area, as these were the points considered to be in contact with a cell. The subset of vessel points was then projected onto the plane of most variance, found using singular value decomposition (Equation 2). The surface area was estimated from this $2 \mathrm{D}$ object by finding its convex hull and then calculating the area of the convex hull using MATLAB. 


$$
d(c, v)=\sqrt{\left(c_{1}-v_{1}\right)^{2}+\left(c_{2}-v_{2}\right)^{2}+\left(c_{3}-v_{3}\right)^{2}}
$$

(Equation 1)

$$
M=U \Sigma V^{*}
$$

(Equation 2)

where $d(c, v)$ is the distance between points $c$ and $v ; c=\left(c_{1}, c_{2}, c_{3}\right)$ is a point of a cell; $v=\left(v_{1}, v_{2}, v_{3}\right)$ is a point of a vessel; $M$ is an $m \times 3$ matrix of the coordinates of the vessel that are in contact with a cell (or points in contact); $U$ is an $m \times m$ orthogonal matrix where $m$ is the number of points in contact; $\Sigma$ is a $m \times 3$ diagonal matrix with the singular values on the diagonal; $V$ is a $3 \times 3$ orthogonal matrix; and $V^{*}$ is the conjugate transpose of the $n S_{n}$ orthogonal matrix $V$.

Lymphatic capillary staining and function analysis. Ears were split along the dorsal-ventral axis and fixed in $4 \%$ paraformaldehyde. Dorsal preparations were stained with goat anti-mouse LYVE-1 Ab (R\&D Systems) followed by Cy3-labeled secondary Ab or, if from Prox 1 reporter mice, visualized directly. Low-magnification images of the lymphatic capillaries near the ear edges were made using a Leica SPE confocal microscope and lymphatic diameter, and branch point density was assessed using Imaris software.

For transport analysis, as previously described (19), the hair from ears was removed 1 day before the experiment. A total of $1 \mu \mathrm{l} \mathrm{Cy5-}$ conjugated dextran (70 kDa; Nanocs) at a concentration of $2 \mathrm{mg} / \mathrm{ml}$ in sterile PBS was injected via a Hamilton syringe intradermally (i.d.) into the ear. Fluorescence was observed through skin using a fluorescence stereomicroscope (M205FA Leica), and images of the skin were acquired each minute for 15 minutes using constant exposure time. Fluorescence intensity and exposure times were adjusted to ensure that intensity values were linearly proportional to the actual fluorescence. Images were processed using ImageJ (http://imagej.nih.gov/ ij/) and Fiji software (http://fiji.sc/Fiji), and the rate of clearance was determined by first calculating the area under the curve of fluorescence intensity in the injection region at each time point, normalized to the initial value. The normalized rate of fluorescence decay was then calculated from the slope of area under the curve versus time. This was considered proportional to the actual rate of Cy5-dextran clearance. Left and right assessments were made at each ear in each mouse, and the 2 normalized values were averaged to generate 1 mean value per mouse per site.

Vessel isolation and cannulation. Mice were anesthetized with pentobarbital sodium (Nembutal, $60 \mathrm{mg} / \mathrm{kg}$, i.p.), and the intestines were exposed through a 2- to $3-\mathrm{cm}$ midline incision. Lymphatic collecting vessels in the mesentery were isolated under a stereomicroscope and placed in Krebs buffer containing 0.5\% BSA. Adipose tissue was carefully removed from isolated lymphatics in a Sylgard-coated chamber. The lymphatic collecting vessels were tied onto 2 glass micropipettes $(\sim 80$ $\mu \mathrm{m}$ OD each) submerged in Krebs buffer in a chamber with a water jacket for temperature control. The perfusion micropipette had 2 lumens; thus, it was capable of perfusing either unlabeled or fluorescently tagged BSA. After cannulation, the vessel was warmed to $37^{\circ} \mathrm{C}$ over 45 minutes on a Zeiss Axiovert 100TV inverted fluorescence microscope.

Lymphatic solute permeability assay. As previously described $(13,42)$, albumin flux $\left(J_{s}, \mathrm{mmol} / \mathrm{s}\right)$ across the lymphatic collecting vessel wall was measured directly with microscope-based photometry. Briefly, lymphatic collecting vessels were surgically excised from the mouse mesentery so that adipose and connective tissues could be carefully removed. These isolated collecting lymphatics were then tied onto glass micropipettes to enable perfusion with solutions containing a fluorescent-tagged albumin or containing an unlabeled albumin. Fluorescence intensity was measured over a rectangular region of interest that sampled light from the vessel lumen and adjacent buffer. All vessels were perfused at a constant, low pressure and flow $\left(<10 \mathrm{~cm} \mathrm{H}_{2} \mathrm{O}\right)$. Upon perfusing the fluorescent albumin, a rapid, step increase in fluorescence intensity $\left(I_{\mathrm{o}}\right)$ was recorded on the photometer. Over time, the fluorescent albumin moved across the lymphatic vessel wall into the surrounding bath solution, which caused a gradual but linear increase in photometer voltage $\left(\mathrm{d} I_{\mathrm{f}} / \mathrm{d} t\right)$. Afterwards, perfusion of the unlabeled albumin washed away all fluorescence, returning the photometer voltage to baseline, and allowed repeated measurements to be made. Albumin permeability $\left(P_{\mathrm{s}}, \mathrm{cm} / \mathrm{s}\right)$ was calculated from a modified form of Fick's first law, which relates albumin flux to a constant (i.e., unchanging) surface area $\left(\mathrm{S}, \mathrm{cm}^{2}\right)$ and concentration gradient $(\Delta \mathrm{C}, \mathrm{mmol} / \mathrm{ml}): P s=J_{s} / S \Delta C=\left(1 / \mathrm{I}_{\mathrm{o}}\right)\left(\mathrm{d} I_{f} / \mathrm{d} t\right)(\mathrm{D} / 4)$, where collecting lymphatic diameter $(D, \mathrm{~cm})$ was simultaneously measured on a computer monitor to ensure that it did not change for the duration of each recording.

Statistics. The statistical significance of differences in mean values was analyzed with Mann-Whitney $U$ test or ANOVA for multiple comparisons, then by Tukey's post hoc test. $P<0.05$ was considered statistically significant. Errors shown in bar graphs and mentioned in text refer to \pm SEM.

Study approval. All experimental procedures were approved by the animal studies committees of Washington University School of Medicine, the Department of Medical Pharmacology and Physiology, University of Missouri, Mount Sinai School of Medicine, Hannover Medical School, or Paris VI University.

\section{Author contributions}

SI, JPS, KW, BTS, KWK, ELK, ACS, EGW, ELG, MJD, BHZ, and GJR designed and executed experiments, analyzed data, and edited the manuscript. MWJ analyzed data. MO, JWW, CB, and PLW executed experiments. MC provided materials. RF, BEI, MJD, $\mathrm{BHZ}$, and GJR supervised experiments and edited the manuscript. SI, JPS, BHZ, and GJR wrote the manuscript.

\section{Acknowledgments}

We thank Theresa and Ken Murphy and Kory Lavine for providing mice and for advice. We also thank Guillermo Oliver (Northwestern University) for provision of Prox1-ERT2-Cre mice. The Advanced Microscopy Facility at the University of Virginia expertly embedded and prepared sections on grids for use in electron microscopy. G.J. Randolph was affiliated with the Mount Sinai School of Medicine (New York, New York, USA) in the early stages of this project. This work was supported by NIH R21 AG046734, R01 AI 049653, Pioneer Award DP1DK109668, and an Innovator Award from the Rainin Foundation (to G.J. Randolph); NIH HL120867 to (M.J. Davis); ERC Advanced Grant GA-322645-Lymphatics-Homing (to R. Förster); NIH R01 HL088554 (to B.E. Isakson); NIH K99 HL124142 and 5T32DK7296 (to J.P. Scallan); and award no. 00056835 from the Fondation de France to (E.L. Gautier). 
Address correspondence to: Gwendalyn J. Randolph or Bernd Zinselmeyer, Department of Pathology and Immunology, Washington University School of Medicine, 425 S. Euclid Avenue, Box 8118, St Louis, Missouri 63110, USA. Phone: 314.286.2345; E-mail: grandolph@path.wustl.edu (G.J. Randolph). Phone:314.362.7464; E-mail: bzinselmeyer@wustl.edu (B. Zinselmeyer).
S. Ivanov's present address is: University of Nice, Nice, France. J.P. Scallan's present address is: University of South Florida, Tampa, Florida, USA. E.L. Kuan's present address is: Immunology Program, Benaroya Research Institute, Seattle, Washington, USA. A.C. Straub's present address is: Department of Pharmacology, University of Pittsburgh, Pittsburgh, Pennsylvania, USA.
1. Randolph GJ, Angeli V, Swartz MA. Dendritic-cell trafficking to lymph nodes through lymphatic vessels. Nat Rev Immunol. 2005;5(8):617-628.

2. Clement CC, Santambrogio L. The lymph selfantigen repertoire. Front Immunol. 2013;4:424.

3. Moussion C, Girard JP. Dendritic cells control lymphocyte entry to lymph nodes through high endothelial venules. Nature. 2011;479(7374):542-546.

4. Kumar V, et al. A dendritic-cell-stromal axis maintains immune responses in lymph nodes. Immunity. 2015;42(4):719-730.

5. Förster R, et al. CCR7 coordinates the primary immune response by establishing functional microenvironments in secondary lymphoid organs. Cell. 1999;99(1):23-33.

6. Ohl L, et al. CCR7 governs skin dendritic cell migration under inflammatory and steady-state conditions. Immunity. 2004;21(2):279-288.

7. Wendland M, et al. Lymph node $\mathrm{T}$ cell homeostasis relies on steady state homing of dendritic cells. Immunity. 2011;35(6):945-957.

8. Weber M, et al. Interstitial dendritic cell guidance by haptotactic chemokine gradients. Science. 2013;339(6117):328-332.

9. Pflicke H, Sixt M. Preformed portals facilitate dendritic cell entry into afferent lymphatic vessels. J Exp Med. 2009;206(13):2925-2935.

10. Lammermann $\mathrm{T}$, et al. Rapid leukocyte migration by integrin-independent flowing and squeezing. Nature. 2008;453(7191):51-55.

11. Hagendoorn J, et al. Endothelial nitric oxide synthase regulates microlymphatic flow via collecting lymphatics. Circ Res. 2004;95(2):204-209.

12. von der Weid PY, Zawieja DC. Lymphatic smooth muscle: the motor unit of lymph drainage. Int $J$ Biochem Cell Biol. 2004;36(7):1147-1153.

13. Scallan JP, Huxley VH. In vivo determination of collecting lymphatic vessel permeability to albumin: a role for lymphatics in exchange. J Physiol. 2010;588(pt 1):243-254.

14. Kuan EL, et al. Collecting lymphatic vessel permeability facilitates adipose tissue inflammation and distribution of antigen to lymph nodehoming adipose tissue dendritic cells. JImmunol. 2015;194(11):5200-5210.

15. Fonseca DM, et al. Microbiota-dependent sequelae of acute infection compromise tissue-specific immunity. Cell. 2015;163(2):354-366.

16. Lindquist RL, et al. Visualizing dendritic cell net- works in vivo. Nat Immunol. 2004;5(12):1243-1250

17. Srinivasan RS, et al. Lineage tracing demonstrates the venous origin of the mammalian lymphatic vasculature. Genes Dev. 2007;21(19):2422-2432.

18. Jakubzick C, et al. Blood monocyte subsets differentially give rise to $\mathrm{CD}_{103^{+}}$and $\mathrm{CD}^{-103^{-}}$pulmonary dendritic cell populations. JImmunol. 2008;180(5):3019-3027.

19. Platt AM, et al. Normal dendritic cell mobilization to lymph nodes under conditions of severe lymphatic hypoplasia. J Immunol. 2013;190(9):4608-4620.

20. Scallan JP, Hill MA, Davis MJ. Lymphatic vascular integrity is disrupted in type 2 diabetes due to impaired nitric oxide signalling. Cardiovasc Res. 2015;107(1):89-97.

21. Ng CP, Hinz B, Swartz MA. Interstitial fluid flow induces myofibroblast differentiation and collagen alignment in vitro. J Cell Sci. 2005; 118(pt 20):4731-4739.

22. Ng CP, Swartz MA. Mechanisms of interstitial flow-induced remodeling of fibroblast-collagen cultures. Ann Biomed Eng. 2006;34(3):446-454.

23. Gibbings D, Befus AD. CD4 and CD8: an insideout coreceptor model for innate immune cells. JLeukoc Biol. 2009;86(2):251-259.

24. Chen Z, et al. CD11c(high)CD8 ${ }^{+}$regulatory $T$ cell feedback inhibits CD4 T cell immune response via Fas ligand-Fas pathway. J Immunol. 2013;190(12):6145-6154.

25. Jung $S$, et al. In vivo depletion of $C D 11 c(+)$ dendritic cells abrogates priming of $\mathrm{CD} 8(+) \mathrm{T}$ cells by exogenous cell-associated antigens. Immunity. 2002;17(2):211-220.

26. Swiecki M, Gilfillan S, Vermi W, Wang Y, Colonna M. Plasmacytoid dendritic cell ablation impacts early interferon responses and antiviral NK and CD8(+) T cell accrual. Immunity. 2010;33(6):955-966.

27. Asselin-Paturel C, Brizard G, Pin JJ, Briere F, Trinchieri G. Mouse strain differences in plasmacytoid dendritic cell frequency and function revealed by a novel monoclonal antibody. JImmunol. 2003;171(12):6466-6477.

28. van Blijswijk J, Schraml BU, Reis e Sousa C. Advantages and limitations of mouse models to deplete dendritic cells. Eur JImmunol. 2013;43(1):22-26.

29. Suzuki S, et al. Critical roles of interferon regulatory factor 4 in $\mathrm{CD} 11 b^{\text {high }} \mathrm{CD} 8 \alpha$-dendritic cell development. Proc Natl Acad Sci U S A. 2004;101(24):8981-8986.

30. Tamura T, et al. IFN regulatory factor- 4 and -8 govern dendritic cell subset development and their functional diversity. J Immunol. 2005;174(5):2573-2581.

31. Schlitzer A, et al. IRF4 transcription factordependent $C D 11 b^{+}$dendritic cells in human and mouse control mucosal IL-17 cytokine responses. Immunity. 2013;38(5):970-983.

32. Klein U, et al. Transcription factor IRF 4 controls plasma cell differentiation and class-switch recombination. Nat Immunol. 2006;7(7):773-782.

33. Edelson BT, et al. Peripheral $\mathrm{CD}_{103}{ }^{+}$dendritic cells form a unified subset developmentally related to $\mathrm{CD} 8 \alpha^{+}$conventional dendritic cells. JExp Med. 2010;207(4):823-836.

34. Persson EK, et al. IRF4 transcription-factordependent $\mathrm{CD} 103(+) \mathrm{CD11b}(+)$ dendritic cells drive mucosal $T$ helper 17 cell differentiation. Immunity. 2013;38(5):958-969.

35. Adair TH, Moffatt DS, Paulsen AW, Guyton AC. Quantitation of changes in lymph protein concentration during lymph node transit. Am J Physiol. 1982;243(3):H351-H359.

36. Adair TH, Guyton AC. Modification of lymph by lymph nodes. II. Effect of increased lymph node venous blood pressure. Am J Physiol. 1983;245(4):H616-H622.

37. Adair TH, Guyton AC. Modification of lymph by lymph nodes. III. Effect of increased lymph hydrostatic pressure. Am J Physiol. 1985; 249(4 pt 2):H777-H782.

38. Lim HY, et al. Hypercholesterolemic mice exhibit lymphatic vessel dysfunction and degeneration. Am J Pathol. 2009;175(3):1328-1337.

39. Mittrucker HW, et al. Requirement for the transcription factor LSIRF/IRF4 for mature $\mathrm{B}$ and $\mathrm{T}$ lymphocyte function. Science. 1997;275(5299):540-543.

40. Zinselmeyer BH, et al. Chapter 16. Two-photon microscopy and multidimensional analysis of cell dynamics. Methods Enzymol. 2009;461:349-378.

41. Zinselmeyer BH, et al. $\mathrm{PD}-1$ promotes immune exhaustion by inducing antiviral $\mathrm{T}$ cell motility paralysis. JExp Med. 2013;210(4):757-774.

42. Scallan JP, Davis MJ, Huxley VH. Permeability and contractile responses of collecting lymphatic vessels elicited by atrial and brain natriuretic peptides. J Physiol. 2013;591(pt 20):5071-5081. 